\title{
A Real-Time Occlusion Aware Hardware Structure for Disparity Map Computation
}

\author{
Christos Georgoulas and Ioannis Andreadis \\ Laboratory of Electronics \\ Department of Electrical and Computer Engineering, Democritus University of Thrace, \\ GR-67 100 Xanthi, Greece \\ \{cgeorg, iandread\} @ee.duth.gr
}

\begin{abstract}
Many machine vision applications deal with depth estimation in a scene. Disparity map recovery from a stereo image pair has been extensively studied by the computer vision community. Previous methods are mainly restricted to software based techniques on general-purpose architectures, presenting relatively high execution time due to the computationally complex algorithms involved. In this paper a new hardware module suitable for real-time disparity map computation module is realized. This enables a hardware based occlusion-aware parallel-pipelined design, implemented on a single FPGA device with a typical operating frequency of $511 \mathrm{MHz}$. It provides accurate disparity map computation at a rate of 768 frames per second, given a stereo image pair with a disparity range of 80 pixels and $640 \times 480$ pixel spatial resolution. The proposed method allows a fast disparity map computational module to be built, enabling a suitable module for real-time stereo vision applications.
\end{abstract}

Keywords: FPGA-hardware implementation, occlusions, real-time imaging, disparity maps, color image processing.

\section{Introduction}

An active area of research is the so-called correspondence problem. It comprises the detection of conjugate pairs in stereo image pairs, i.e. to find for each point in the left image, its corresponding in the right image [1]. The candidate matching points should be distinctly different from their surrounding pixels. Even two identical cameras have different sensor parameters, which introduces pixel intensity value variations for the same scene point. Thus, suitable feature extraction should precede stereo matching. The two major categories of feature extraction algorithms are: area-based $[2,3]$ and feature-based [4, 5]. Area-based algorithms use local pixel intensities as a distance measure and they produce dense disparity maps, i.e. process the whole area of the image pair. On the other hand, feature-based algorithms rely on certain points of interest. These points are selected according to appropriate feature detectors. Comparing the strengths and weaknesses of these two major categories, the main advantage of feature-based compared to area-based algorithms, is that they produce more accurate results. The disadvantages are that they are much slower and produce sparse disparity 
maps [4]. This makes area-based algorithms suitable for real-time applications [6]. A major problem though with area-based algorithms is that the computed disparity map contains an amount of false matched points due to uncertainty during the disparity selection stage, especially within featureless regions or at occlusion boundaries. This uncertainty cannot be easily handled by dense stereo algorithms. The occlusion problem in stereovision refers to the fact that some scene points are visible to one camera but not the other, due to the geometry of the scene and camera setup geometries. Many researches have focused on detecting and measuring occlusion regions in stereo imagery, and recovering accurate depth estimates for these regions, as evaluated in [7].

In our later work [8], a three-stage module addressing the stereo vision matching problem aimed at real-time applications was proposed and implemented in hardware. It employed an adaptive window constrained search as well as a Cellular Automata (CA) approach, as a post-processing step, for efficient false reconstructions removal, improving the accuracy of the resulting disparity maps. The work was further extended [9], implementing a Fuzzy Inference System (FIS) hardware structure, as a post processing step for performance and accuracy improvement.

In this paper, a novel three stage real-time hardware implemented disparity map computation module is proposed, extending our previous work [8,9], both from qualitative and quantitative terms, concerning the quality of the produced disparity map and the frame rate output of the module. The proposed module can efficiently deal with speed demanding applications since disparity maps are computed at a rate of 768 frames per second for a $640 \times 480$ pixel resolution stereo pair. Its main advantage compared to previous algorithms is its high processing speed, which is crucial in realtime practical applications such as three-dimensional modeling, vision-guided mobile robots, object recognition and navigation, biometrics and many more. To eliminate false correspondences, an occlusion-aware module was implemented, concerning both left-right and right-left disparity consistency information. The above requires the initial computation of two disparity maps, in order to retrieve the inconsistent disparities, which comprise to occluded areas.

The paper is organized as follows: the proposed algorithm is described in Section 2, the proposed hardware structure is presented in Section 3, experimental results are shown in Section 4 and conclusions are provided in Section 5.

\section{Proposed Algorithm}

The disparity maps are initially generated with the use of Sum of Absolute Differences (SAD). Afterwards, the occluded areas and other false matched points are extracted from the previously calculated maps, to finally refine and obtain the resulting disparity maps. All three steps are fully implemented in hardware on an FPGA device.

\subsection{Color SAD Window Based Technique}

A color SAD window based algorithm was used for the disparity map generation. As in our later work $[8,9]$, this choice was made to increase speed. Sum of squared 
differences, correlation, or image moments, could also be used, but were not selected after considering speed issues, since greater computational complexity is involved in these metrics, enhancing their required processing times compared to SAD. Moreover, the SAD can be directly implemented in hardware, due to its simplicity. The $\mathrm{SAD}$ equations used can be seen below:

$$
\begin{aligned}
& S A D(i, j, d)_{\text {left-right }}=\sum_{\mu=-w}^{w} \sum_{v=-w}^{w} \sum_{k=1}^{3}\left|I_{l}(i+\mu, j+v, k)-I_{r}(i+\mu, j-d+v, k)\right|, \\
& S A D(i, j, d)_{\text {right-left }}=\sum_{\mu=-w}^{w} \sum_{v=-w}^{w} \sum_{k=1}^{3}\left|I_{l}(i+\mu, j+d+v, k)-I_{r}(i+\mu, j+v, k)\right|,
\end{aligned}
$$

where $w$ is the square window size fixed to $7 \times 7$ pixels, $I_{l}$ and $I_{r}$ denote the left and right image pixel grayscale values, $d$ is the disparity range, and $i, j, k$ are the coordinates (rows, columns, color component) of the center pixel of the window for which the SAD is computed.

Once the two SADs are computed for all pixels and for all disparity values, a similarity accumulator has been constructed for each pixel, for each consistency, which indicates the most likely disparity. A search in the SADs for all disparity values, $\left(d_{\min }\right.$ up to $\left.d_{\max }\right)$, is performed for every pixel, and at the disparity index where the SADs are minimum, these values are given as the corresponding pixel values for the left-right and right-left disparity maps:

$$
D(i, j)=\underset{d \in\left[d_{\min }, d_{\max }\right]}{\arg \min } \operatorname{SAD}(i, j, d) .
$$

\subsection{Occlusion and False Matching Detection}

There are three main classes of algorithms for handling occlusions: 1) methods that detect occlusions $[10,11], 2)$ methods that reduce sensitivity to occlusions $[12,13]$, and 3) methods that model the occlusion geometry [14, 15]. Considering the first class, left-right consistencychecking may also be used to detect occlusion boundaries. Computing two disparity maps, one based on the correspondence from the left image to the right image, and the other based on the correspondence from the right image to the left image, inconsistent disparities are assumed to represent occluded regions in the scene. Left-right consistency checking is also known as the "two-views constraint". This technique is well suited to remove false correspondences caused by occluded areas within a scene [11]. Due to its simplicity and overall good performance, this technique was implemented in many real-time stereo vision systems [16-18].

Disparity maps are usually corrupted due to false correspondence matches. There might be occluded areas in the scene, leading to false disparity assignment. Using the left-right consistency checking, valid disparity values are considered, only those that are consistent in both disparity maps, i.e. those that do not lie within occluded areas. A pixel that lies within an occluded area will have different disparity value in the left 
disparity map, from its consistent pixel in the right disparity map. For example, a nonoccluded pixel in the left disparity image must have a unique pixel with equally assigned disparity value in the right disparity map according to the following equations:

$$
\begin{aligned}
& D_{\text {left-right }}(i, j)=D_{\text {right-left }}(i, j-d),\left(d=D_{\text {left-right }}(i, j)\right), \\
& D_{\text {right-left }}(i, j)=D_{\text {left-right }}(i, j+d),\left(d=D_{\text {right-left }}(i, j)\right) .
\end{aligned}
$$

The same applies, for false matched points not exclusively due to occlusions, but due to textureless areas or sensor parameter variations. These points are assigned with a false disparity value during the disparity map assignment stage described by equation (3), since there might be more than one minimum SAD value for a given pixel, which leads to false disparity value assignment for that pixel. Thus, the disparity value assigned to some pixels does not correspond to the appropriate correct value.

Performing this consistency check, the occluded pixel along with the false matched points within the scene can be derived. These are depicted in Figure 1, (a) for leftright and (b) for right-left disparity map respectively.

\subsection{Disparity Refinement}

Once the occluded areas along with the false matched points have been extracted, the disparity maps are refined in order to improve their resulting quality. Considering the occluded and false matched pixels in the left-right disparity map, their value is being replaced by one of their neighboring pixels in the range $(i, j-5)$ up to $(i, j-1)$ according to which approximates best equation (4), after being crossed-checked with right-left disparity map corresponding pixels. In the case that the neighboring pixels are unmatched, disparity refinement cannot be performed for the given pixels. The proposed method thus yields semi-dense disparity results. The neighborhood size has been selected after a series of tests under various size configurations, to obtain adequate performance [19]. The right-left disparity map is refined in the similar manner according to equation (5). This improves the resulting disparity maps accuracy, since uncertainty can be eliminated by an appropriate disparity value assignment.

\section{Hardware Description}

The module implementation relies on the use of an FPGA device. Considering their flexibility in terms of reconfigurability and ease of use convenience, FPGAs

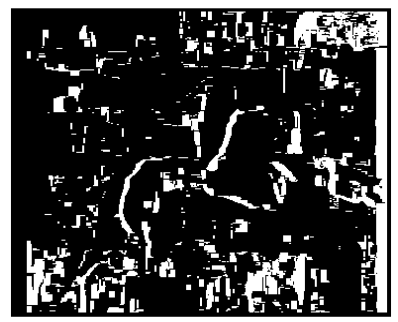

(a)

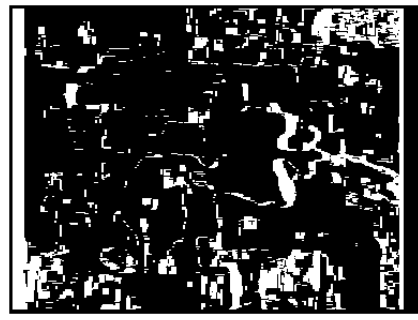

(b)

Fig. 1. Occluded and false matched pixels 
have become very popular in digital systems synthesis, providing high performance at a reasonable price on digital system design [20]. The module was implemented in parallel pipelined architecture, realized on a single FPGA device of the Stratix IV family of Altera Devices. The typical operating clock frequency was found to be 511 MHz. The block diagram of the proposed module can be seen in Figure 2.

$\mathrm{SAD}$ calculation is required for a $7 \times 7$ pixel size window, throughout the whole image. 24-bit color depth images are used as inputs. The calculated disparity values are 7-bit binary words, since the proposed module was designed to operate for up to 80 disparity levels, $\left(2^{7}=128\right.$ different levels $)$.

Two serpentine-type input register arrangements are used to temporally store the left and right image working windows. The serpentine input blocks are considered in order to increase the processing speed of the proposed module, since overlapping pixels usually exist between adjacent windows in window-based operations. This architecture is used to temporarily store overlapping pixels in order to reduce the clock cycles needed to load image pixels into the module. After an initial latency period which depends on the image size, output is provided once every clock cycle.

Two similar sets of registers are used to store $d_{\max } 3 \times 7 \times 7$ working windows for the left and right image $\mathrm{R}, \mathrm{G}$ and $\mathrm{B}$ components. The number of the registers concerning the image inputs, is proportional to the number of the disparity range; i.e. for a disparity range up to 80 and a working window of $7 \times 7,[7 \times 7+((80-1) \times 7)]=602$ registers are needed for each color component for each image input. A disparity selection input pin is used to enable the required number of registers according to the given disparity range, to increase the speed performance when operating at smaller disparity ranges, concerning the time needed to initially fill the required registers sizes with the image pixel values. The SADs for left-right and right-left disparity maps are computed according to equations (1) and (2). The disparity value, $\left(\mathrm{d}_{\text {min }}\right.$ up to $d_{\max }$ ), for which the SAD is minimum for each pixel, equation (3), is selected as the corresponding pixel value for disparity map, which is then fed to the occlusion/false match detector circuit.

A range of $\mathrm{d}_{\max }$ disparity values is required by the occlusion/false match detector module. This is due to the fact that consistency checking is performed for all possible horizontal displacements according to equations (4) and (5). Therefore, for the case of an operating range of 80 disparity levels, for the left-right disparity map check, the $\mathrm{D}_{\text {left-right }}(\mathrm{i}, \mathrm{j})$ disparity value is checked against its unique consistent match which lies in the $D_{\text {right-left }}(i, j-80$ up to $j$ ) range. Every pixel is checked against its consistent match, and if equations (4) and (5) are not satisfied, the pixel is marked as occluded or false assigned. To enable such operation a set of 80 registers is used for each map, to store the necessary disparity values calculated from previous module stages.

Finally the disparity map refinement is performed by obtaining the occluded and false assigned pixels from previous stage and replacing their values considering the neighboring $(\mathrm{i}, \mathrm{j}-5)$ up to $(\mathrm{i}, \mathrm{j}-1)$ pixel range. A set of registers is used to store the data required to perform the disparity value replacement. A total of $5 \times 7$ bits are required from each consistency, which are fed into the disparity map refinement unit from the previous stage. Once the refinement is performed, the final 7-bit disparity value for both left-right and right-left consistency is obtained at the module output. 


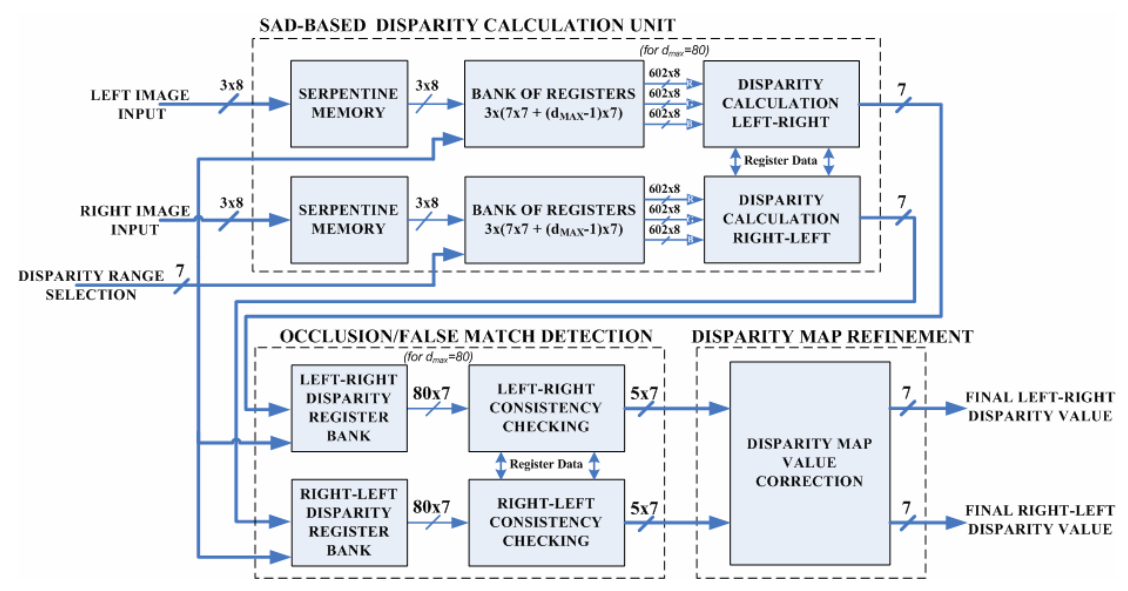

Fig. 2. Block diagram of the proposed module

The architecture presented in Section 3 has been implemented using Quartus II schematic editor by Altera. It has been then simulated to prove functionality, and once tested, finally mapped on an FPGA device. The analytical specifications of the target device are shown in Table 1.

\section{Experimental Results}

The proposed occlusion and false assignment detection provides satisfactory improvement in the accuracy of the resulting disparity maps, while preserving all the necessary details of the disparity map depth values. The resulting disparity maps are presented in Figure 3 along with original image pairs for (a) Tsukuba and (b) Cones.

The hardware implemented module enables an appropriate method in real-time demanding applications. The relationship between the number of frames processed per second and the processed image size, for an operating range of 80 disparity levels, is presented in Table 2. As it can be observed, the module presents real-time response even for high resolution images.

Compared to existing methods in terms of speed, it presents higher processing rates. Quantitative results of the proposed module under various configurations can be seen in Table 3, and compared to our previous methods [8,9] and other existing methods [21-25], in Table 4. Compared to [8, 9,21-25], our method proves its performance, as it can be seen in Table 4. The Acc (accuracy) term, shown in Tables 3 and 4 , states the ratio of the pixels given a correct disparity value (as compared with the ground truth) to the total assigned pixels, where the Cov (coverage) term states the percentage of the image total pixels, for which a disparity value has been assigned. The total accuracy (Tot Acc) term, shown in Table 4, is the percentage of the image total pixels, for which a correct disparity value has been assigned. The standard Nonocc, All and Disc parameters, that appear in Middlebury stereo evaluation webpage (http://vision.middlebury.edu/stereo/), were not followed since they correspond to dense disparity maps metrics, whereas the proposed method yields semi-dense results. 

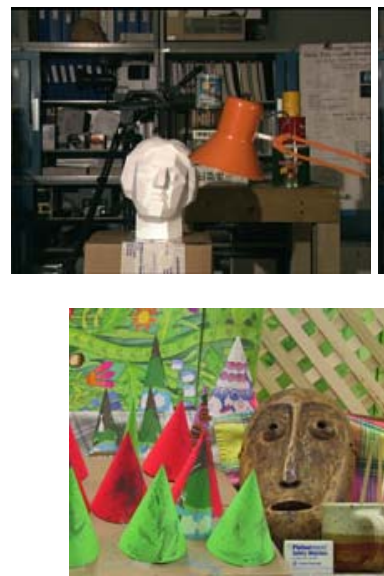

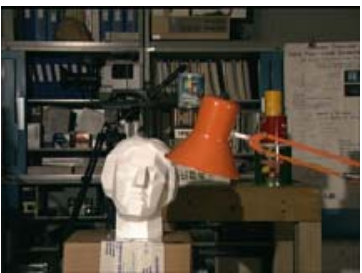

(a)

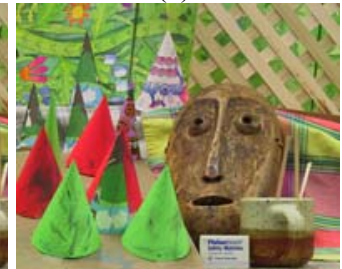

(b)
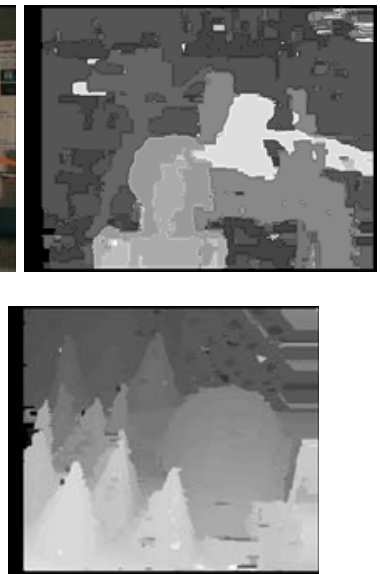

Fig. 3. Resulting disparity maps along with original image pairs for (a) Tsukuba (b) Cones

Table 1. Specifications of target device

\begin{tabular}{|c|c|c|c|c|c|}
\hline Device & Block memory bits (\%) & Registers & ALUTs (\%) & LABs (\%) & Pins (\%) \\
\hline $\begin{array}{c}\text { EP4SGX290 } \\
\text { HF35C2 }\end{array}$ & $\begin{array}{c}<1 \\
(192 / \\
13,934,592) \\
\end{array}$ & 15,442 & $\begin{array}{c}59 \\
(143,653 / \\
244,160) \\
\end{array}$ & $\begin{array}{c}74 \\
(9,036 \\
/ 12,208) \\
\end{array}$ & $\begin{array}{c}10 \\
(70 / 660)\end{array}$ \\
\hline
\end{tabular}

Table 2. Frame rate output of realized module

\begin{tabular}{|c|c|c|c|c|c|}
\hline Disp. levels & \multicolumn{5}{|c|}{80} \\
\hline $\begin{array}{c}\text { Image size } \\
\text { (pixels) }\end{array}$ & $320 \times 240$ & $640 \times 480$ & $800 \times 600$ & $1024 \times 768$ & $1280 \times 1024$ \\
\hline Frames / sec & 2042 & 768 & 550 & 375 & 251 \\
\hline
\end{tabular}

The accuracy achieved by the proposed method along with the speed performance under various levels of disparity, enable an efficient module that can perform adequately enough in stereo vision applications, regarding less than $10 \%$ decrease in accuracy and more than a hundred times faster processing speed compared to previous algorithms [21-25], Table 4. Moreover, the proposed module exhibits real time speed response preservation even at practical stereo vision large disparity ranges, while other methods [21-25] present an exponential rise in time needed to compute larger disparity ranges. This can be seen in Table 4.

Compared to other FPGA-based approaches, with practical stereo image data, the proposed method presents higher processing rates. In [26], an FPGA-based module reaches 10 frames per second for a sub-sampled 640x480 pixel resolution image with a disparity range of 30 levels. In [27], a 3D-vision system implemented for tracking people, reaches 30 frames per second for a $512 \times 480$ pixel resolution image pair with 52 
levels of disparity. In [28], an FPGA-based system achieves 19 frames per second for a 640x480 image pair resolution with 80 levels of disparity. Additionally in [29], a performance of 600 frames per second is reported, for a 450x375 pixel resolution image pair with a disparity range of 100 levels. The proposed method performs higher processing rates compared to [26-29], reaching 768 frames per second for a $640 \times 480$ pixel resolution image pair with 80 levels of disparity, as it can be observed in Table 2 .

Finally, it must be noted that the proposed module results to semi-dense disparity estimation. The depth information loss due to the semi-dense results can be adequately balanced by the high output rate, since much more depth estimations are being carried out, and more importantly in real-time speeds. On the other hand, dense stereo algorithms are designed to perform better in the accuracy term, and to present much slower output rates. This occurs due to their design architecture complexity, that involves composite calculations and to their sometimes required repetitive behaviour to reach such highly accurate results.

Table 3. Quantitative results of the proposed module under various configurations

\begin{tabular}{|c|c|c|c|c|c|c|c|c|}
\hline & \multicolumn{2}{|c|}{ Tsukuba } & \multicolumn{2}{|c|}{ Venus } & \multicolumn{2}{|c|}{ Cones } & \multicolumn{2}{|c|}{ Teddy } \\
\hline & $\begin{array}{l}\text { Acc } \\
(\%)\end{array}$ & $\begin{array}{l}\operatorname{Cov} \\
(\%)\end{array}$ & $\begin{array}{l}\text { Acc } \\
(\%)\end{array}$ & $\begin{array}{l}\text { Cov } \\
(\%)\end{array}$ & $\begin{array}{l}\text { Acc } \\
(\%)\end{array}$ & $\begin{array}{l}\text { Cov } \\
(\%)\end{array}$ & $\begin{array}{l}\text { Acc } \\
(\%)\end{array}$ & $\begin{array}{l}\text { Cov } \\
(\%)\end{array}$ \\
\hline Initial Disparity Map & 94 & 77 & 93 & 75 & 99 & 80 & 98 & 77 \\
\hline Refined Disparity Map & 95 & 91 & 95 & 92 & 94 & 93 & 92 & 95 \\
\hline
\end{tabular}

Table 4. Quantitative results of the proposed module compared to previous algorithms

\begin{tabular}{|c|c|c|c|c|c|c|c|c|c|c|c|c|}
\hline & \multicolumn{4}{|c|}{$\begin{array}{c}\text { Tsukuba }(384 \times 288, \\
\text { disp. levels }=16)\end{array}$} & \multicolumn{4}{|c|}{$\begin{array}{l}\text { Venus }(434 \times 383, \\
\text { disp. levels }=20)\end{array}$} & \multicolumn{4}{|c|}{$\begin{array}{l}\text { Cones }(450 \times 375, \\
\text { disp. } \text { Levels }=60)\end{array}$} \\
\hline & $\begin{array}{l}\text { Acc } \\
(\%)\end{array}$ & $\begin{array}{l}\text { Cov } \\
(\%)\end{array}$ & $\begin{array}{l}\text { Tot } \\
\text { Acc } \\
(\%)\end{array}$ & $\begin{array}{l}\text { Time } \\
(\mathrm{ms})\end{array}$ & $\begin{array}{l}\text { Acc } \\
(\%)\end{array}$ & $\begin{array}{l}\text { Cov } \\
(\%)\end{array}$ & $\begin{array}{l}\text { Tot } \\
\text { Acc } \\
(\%)\end{array}$ & $\begin{array}{l}\text { Time } \\
(\mathrm{ms})\end{array}$ & $\begin{array}{l}\text { Acc } \\
(\%)\end{array}$ & $\begin{array}{l}\text { Cov } \\
(\%)\end{array}$ & $\begin{array}{l}\text { Tot } \\
\text { Acc } \\
(\%)\end{array}$ & $\begin{array}{l}\text { Time } \\
\text { (ms) }\end{array}$ \\
\hline [8] & 88 & 51 & 44.88 & 1.3 & 92 & 69 & 63.48 & 1.97 & 72 & 56 & 40.32 & 2 \\
\hline [24] & 99.64 & 75 & 74.73 & 6,000 & 99.84 & 73 & 72.88 & 13,000 & - & 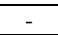 & - & - \\
\hline [25] & 99.68 & 76 & 75.76 & 62 & 99.82 & 73 & 72.87 & 156 & 99.21 & 55 & 54.56 & 328 \\
\hline [9] & 89 & 92 & 81.88 & 0.8 & 92 & 90 & 82.80 & 1.21 & 99 & 93 & 92.07 & 1.26 \\
\hline [21] & 99.7 & 85 & 84.75 & 42 & 99.79 & 86 & 85.82 & 109 & - & - & - & - \\
\hline Proposed & 95 & 91 & 86.45 & 0.29 & 95 & 92 & 87.40 & 0.44 & 92 & 95 & \begin{tabular}{|l|l|}
87.4 \\
\end{tabular} & 0.33 \\
\hline [23] & 99.47 & 93 & 92.51 & 178 & 98.91 & 93 & 91.99 & 216 & 95.02 & 78 & 74.11 & 293 \\
\hline [22] & 99.76 & 95.2 & 94.97 & 4,400 & 99.91 & 92.9 & 92.82 & 11,100 & - & 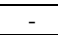 & - & - \\
\hline
\end{tabular}

(-) Not Provided

\section{Conclusions}

This paper presents a hardware implemented three-stage module, addressing the stereo vision matching problem aimed at real time applications, which extends our previous implemented algorithms $[8,9]$. An SAD window based technique using full color RGB images as well as an occlusion detection approach to remove false matchings are 
employed. The architecture is based on fully parallel-pipelined blocks in order to achieve maximum processing speed. Depending on the required operational disparity range, the proposed module can be parameterized, to adapt to the given configuration. Both from qualitative and quantitative terms, concerning the quality of the produced disparity map and the frame rate output of the module, a highly efficient method dealing with the stereo correspondence problem has been proposed. Real-time speeds rated up to 768 frames per second for a $640 \times 480$ pixel resolution image pair with 80 disparity levels, are achieved, which enable the proposed module for real stereo vision applications. The proposed module maintains almost constant frame rate output, even for large disparity ranges, considering that disparity levels in most practical stereo vision applications range from 60 up to 100.Its maximum disparity operating range is designed up to 80 levels, but it can be straightforwardly implemented even further, to enable even larger disparity ranges.

Additionally, the proposed module design was targeted on a single Altera FPGA device. As a result, it could be applied to enable an efficient system including threedimensional modeling, vision-guided mobile robots, object recognition and navigation, biometrics and many more. The proposed analysis and results confirm that semidense disparity maps can be efficiently calculated at real-time rates, whereas other methods have proved that the improvement in the disparity map quality results in speed reduction. This trade-off will always be present in this type of implementations. The proposed module can effectively be applied to practical real-time stereo vision applications due to its speed performance.

\section{References}

1. Barnard, S.T., Thompson, W.B.: Disparity analysis of images. IEEE Transactions on Pattern Analysis and Machine Intelligence 2, 333-340 (1980)

2. Di Stefano, L., Marchionni, M., Mattoccia, S.: A fast area-based stereo matching algorithm. Image and Vision Computing 22(12), 983-1005 (2004)

3. Muhlmann, K., Maier, D., Hesser, J., Manner, R.: Calculating dense disparity maps from color stereo images, an efficient implementation. Int. J. Comput. Vision 47(1-3), 79-88 (2002)

4. Jordan, J.R., Bovik, A.C.: Using chromatic information in edge-based stereo correspondence. Computer Vision, Graphics, and Image Processing: Image Understanding 54(1), 98-118 (1991)

5. Baumberg, A.: Reliable feature matching across widely separated views. In: Proceedings of the IEEE Conference on Computer Vision and Pattern Recognition, pp. 774-781 (2000)

6. Hirschmuller, H.: Improvements in Real-Time Correlation-Based Stereo Vision. In: Proceedings of IEEE Workshop on Stereo and Multi-Baseline Vision, pp. 141-148 (2001)

7. Brown, M., Burschka, D., Hager, G.: Advances in computational stereo. IEEE Transactions on Image Processing 25, 993-1008 (2003)

8. Georgoulas, C., Kotoulas, L., Sirakoulis, G., Andreadis, I., Gasteratos, A.: Real-Time Disparity Map Computation Module. Journal of Microprocessors \& Microsystems 32(3), 159-170 (2008)

9. Georgoulas, C., Andreadis, I.: A real-time fuzzy hardware structure for disparity map computation. Submitted to Journal of Real-Time Image Processing 
10. Chang, C., Chatterjee, S., Kube, P.R.: On an Analysis of Static Occlusion in Stereo Vision. In: Proceedings of Computer Vision and Pattern Recognition, pp. 722-723 (1991)

11. Fua, P.: A Parallel Stereo Algorithm that Produces Dense Depth Maps and Preserves Image Features. Machine Vision and Applications 6(1), 35-49 (1993)

12. Bhat, D.N., Nayar, S.K.: Ordinal Measures for Image Correspondence. IEEE Transactions on Pattern Analysis and Machine Intelligence 20(4), 415-423 (1998)

13. Sara, R., Bajcsy, R.: On Occluding Contour Artifacts in Stereo Vision. In: Proceedings of Computer Vision and Pattern Recognition, pp. 852-857 (1997)

14. Belhumeur, P.N.: A Bayesian Approach to Binocular Stereopsis. Int. J. Computer Vision 19(3), 23-260 (1996)

15. Birchfield, S., Tomasi, C.: Depth Discontinuities by Pixel-to- Pixel Stereo. In: Proceedings of the IEEE International Conference on Computer Vision, pp. 1073-1080 (1998)

16. Faugeras, O., et al.: Real-time correlation-based stereo: algorithm, implementations and application. Technical Report RR 2013, INRIA (1993)

17. Konolige, K.: Small vision systems: Hardware and implementation. In: $8^{\text {th }}$ International Symposium on Robotics Research, Hayama, Japan, pp. 203-212. Springer, London (1997)

18. Matthies, L., Kelly, A., Litwin, T.: Obstacle detection for unmanned ground vehicles: A progress report. In: International Symposium of Robotics Research, Munich, Germany (1995)

19. Scharstein, D., Szeliski, R.: A taxonomy and evaluation of dense two-frame stereo correspondence algorithms. Int. J. Comput. Vision 47(1-3), 7-42 (2002)

20. Leeser, M., Hauck, S., Tessier, R.: Editorial: field-programmable gate arrays in embedded systems. EURASIP Journal on Embedded Systems (1), 11 (2006)

21. Gong, M., Yang, Y.H.: Near real-time reliable stereo matching using programmable graphics hardware. In: Proceedings of IEEE Conference on Computer Vision and Pattern Recognition, pp. 924-931 (2005)

22. Kim, J.C., Lee, K.M., Choi, B.T., Lee, S.U.: A dense stereo matching using two-pass dynamic programming with generalized ground control points. In: Proceedings of the IEEE Conference on Computer Vision and Pattern Recognition, pp. 1075-1082 (2005)

23. Gong, M., Yang, Y.H.: Real-Time Stereo Matching Using Orthogonal Reliability-Based Dynamic Programming. IEEE Transactions on Image Processing 16(3), 879-884 (2007)

24. Veksler, O.: Extracting Dense Features for Visual Correspondence with Graph Cuts. In: Proceedings of IEEE Conference on Computer Vision and Pattern Recognition, pp. 689-694 (2003)

25. Gong, M., Yang, Y.H.: Fast Unambiguous Stereo Matching Using Reliability-Based Dynamic Programming. IEEE Transactions on Pattern Analysis and Machine Intelligence 27(6), 998-1003 (2005)

26. Khaleghi, B., Ahuja, S., Wu, J.Q.M.: A new Miniaturized Embedded Stereo-Vision System (MESVS-I). In: Proceedings of Canadian Conference on Computer and Robot Vision, pp. 26-33 (2008)

27. Woodill, J.I., Buck, R., Jurasek, D., Gordon, G., Brown, T.: 3D Vision: Developing an Embedded Stereo-Vision System. IEEE Computer 40(5), 106-108 (2007)

28. Miyajima, Y., Maruyama, T.: A Real-Time Stereo Vision System with FPGA. In: Y. K. Cheung, P., Constantinides, G.A. (eds.) FPL 2003. LNCS, vol. 2778, pp. 448-457. Springer, Heidelberg (2003)

29. Ambrosch, K., Humenberger, M., Kubinger, W., Steininger, A.: SAD-based Stereo Matching Using FPGAs. In: Embedded Computer Vision part II, pp. 121-138. Springer, London (2009) 